\section{Autologe Transfusion bei Skolioseoperationen: Präoperative Eigenblutspende und intraoperative maschinelle Autotransfusion}

\author{
E. Hansen*, B.Pollwein ${ }^{*}$, E.Martin*, M.U. Heim**, \\ S. Horst**, K. A. Matzen ${ }^{* * *}$, K. Peter
}

* Institut für Anästhesiologie (Direktor: Prof. Dr. K. Peter)

*: Transfusionszentrum III. Med. Klinik/BSB-BRK

(Leiter: Prof. Dr. W. Mempel)

$* * *$ Orthopädische Klinik und Poliklinik (Direktor: Prof. Dr. M. Jäger†) der Universität München am Klinikum Großhadern

Die dorsale Spondylodese mit dem Instrumentarium nach Harrington stellt eine effektive Behandlungsmethode idiopathischer Skoliosen dar(19). Risiken entstehen dabei nicht zuletzt durch den hohen intraoperativen Blutverlust, der regelmäßig zur Transfusion von homologem Blut führt $(14,15,17,23)$. Mit der homologen Transfusion gehen erhebliche Nachteile einher, wie Lagerungsschäden des Blutes oder eine Sensibilisierung gegen Blutgruppen-, Protein- und Gewebsantigene, und erhebliche Risiken, wie Infektionsübertragung oder Unverträglichkeitsreaktionen $(13,20)$. Eine wirksame Möglichkeit den Bedarf an Fremdblut und die damit verbundenen Gefahren zu verringern stellt die autologe Transfusion dar $(6,25,30)$. Sie bedeutet die Gabe von patienteneigenem Blut. Unterschieden nach Art und Zeitpunkt der Blutgewinnung sind folgende Verfahren autologer Transfusion möglich:

präoperativ Eigenblutspende

perioperativ normovolämische Hämodilution

intraoperativ maschinelle Autotransfusion

postoperativ Drainagenblutretransfusion

Bei der präoperativen Eigenblutspende wird dem Patienten zu festgelegten Zeitpunkten vor einem elektiven Eingriff durch Venenpunktion eine Blutkonserve abgenommen. Die physiologische Reaktion auf eine derartige Blutspende umfaßt den Ausgleich des Blutvolumens durch Erhöhung des Plasmavolumens innerhalb von 48 Stunden und eine Erythrozytenneubildung, die nach etwa 5 Tagen wirksam wird und ihr Maximum um den 10. Tag erreicht. Bei ausreichender Eisensubstitution erhöht sich die Erythropoese auf das 3-5fache und erlaubt fortgesetzte, wöchentliche Blutabnahmen ohne Abfall des Hämoglobinwertes (7). Erfahrungen zeigen, daß Blutabnahmen auch bei nicht-typischen Blutspendern wie älteren Patienten, Schwangeren, Koronarkranken oder Kindern möglich sind $(8,16)$.

Bei konventioneller Lagerung bei $4^{\circ} \mathrm{C}$ ist die Zahl von Eigenblutkonserven mit vertretbarer Lagerungsdauer zum Operationstermin und mit ausreichenden Perioden zwischen den Spenden jedoch auf 2-3 begrenzt. Durch teil-

\section{Zusammenfassung}

Autologe Transfusion wurde eingesetzt, um den hohen Blutbedarf bei Skolioseoperationen zu senken. Die Einsparung etwa der Hälfte des Blutverlustes durch intraoperative Autotransfusion (35 Patienten) führte zu einer entsprechenden Reduktion an homologer Transfusion. Erst durch die Kombination von präoperativer Eigenblutspende und intraoperativer Autotransfusion (37 Patienten) konnte die Fremdblutgabe bei vielen $\mathrm{Pa}$ tienten völlig vermieden werden. Tiefgefrierung machte die Blutspende unabhängig von der Lagerungsdauer und ermöglichte es, auch den Plasmabedarf autolog zu decken. Transfusionsrisiken werden damit umgangen.

Autologous Transfusion in Scoliosis Surgery: Preoperative Blood Donation and Intraoperative Autotransfusion

Autologous transfusion was used to reduce the high demand for blood accompanied with scoliosis surgery. Half of the blood loss could be saved by intraoperative autotransfusion ( 35 patients). This resulted in a corresponding reduction in homologous transfusion. An elimination of the need for homologous blood could be achieved only by the combination of preoperative blood donation and intraoperative autotransfusion (37 patients). With freezing the predeposit blood was independent of storage time and autologous plasma was availabe. Thus, the risks of transfusion can be avoided.

weise Rücktransfusion gelagerten Eigenblutes bei der darauffolgenden Spende, der aufwendigen „leap-frog“-Methode, läßt sich diese Zahl etwas erhöhen (3). Von dem bei einer Spende abgenommenen Blut kann durch Zentrifugation das Plasma abgetrennt und als fresh-frozen-Plasma (FFP) eingefroren gelagert werden. Die Möglichkeit, auch Erythrozyten einzugefrieren, macht die Eigenblutspende von der Lagerungsdauer unabhängig(31). Zudem sind Erythrozyten nach Tiefkühlkonservierung in ihrer Funktionsfähigkeit und ihrer Überlebensdauer frischen Zellen vergleichbar und damit konventionellen Blutkonserven weitaus überlegen (31).

Patienten mit idiopathischer Skoliose sind gewöhnlich lange vor ihrer Operation in Behandlung und daher sehr geeignet für die Planung eines Blutspendeprogramms. Durch ihr Alter und ihre Grunderkrankung, mit Einschränkung der kardialen und pulmonalen Leistungsfähigkeit, sind sie jedoch nur begrenzt mit Blutspendern zu vergleichen. Die bisherigen Erfahrungen mit Eigenblutspende für Skolioseoperationen $(8,15,17,32)$ ergaben keine wesentlichen $\mathrm{Ne}$ benwirkungen. Wegen der konventionellen Lagerung des Vollblutes bei $4{ }^{\circ} \mathrm{C}$ waren in diesen Studien maximal drei Blutabnahmen in kurzem Abstand möglich, und ein leichter präoperativer Abfall des Hämatokrits mußte in Kauf genommen werden.

Bei der akuten, normovolämischen Hämodilution wird unmittelbar vor Operationsbeginn eine Blutabnahme unter gleichzeitigem Volumenersatz mit kolloidalen Lösungen 
durchgeführt. Die Verringerung der Sauerstoffträger führt nicht zu einer Hypoxie, solange das Blutvolumen aufrecht erhalten bleibt. Durch die verminderte Blutviskosität kommt es nämlich über eine Abnahme des peripheren $\mathrm{Ge}$ fäßwiderstandes zu einem erhöhten venösen Rückstrom und bei gleichbleibender Herzfrequenz zu einem Anstieg des Herzminutenvolumens. Bis zu einer Blutverdünnung auf einem Hämatokrit von $25 \%$ ist dadurch nachweislich eine ausreichende Sauerstoffversorgung der Gewebe gewährleistet (29). Normovolämie ist die unabdingbare Voraussetzung für diese Adaptation an die Verdünnungsanämie. Sie läßt sich nur unter Verwendung von langwirksamen Kolloiden, wie Dextran-, Hydroxyäthylstärke- oder Humanalbuminlösungen, sicher aufrecht erhalten. Eine weitere Vorbedingung ist die kardiale Leistungsfähigkeit zur Erhöhung des Schlagvolumens.

Der intraoperative Verlust von verdünntem Blut und die Rücktransfusion des vollwertigen Eigenwarmblutes am Ende der Operation kann zur Einsparung von 1-2 Transfusionseinheiten führen(18). Zusätzlich bewirkt die Hämodilution eine Verbesserung der Mikrozirkulation und eine Thromboseprophylaxe. Die Bedeutung der Hämodilution liegt heute vor allem in der Anwendung ihres Prinzips in zahlreichen klinischen Situationen, z. B. der Schocktherapie, und in der Erkenntnis, niedrigere Hämatokritwerte bei Normovolämie tolerieren zu können.

Eine deutliche Bluteinsparung bei Skolioseoperationen wurde nur mit extremer Hämodilution bis auf Hämatokritwerte von $15 \%$ erreicht (17). Diese Form der Methode erfordert jedoch ein invasives Monitoring und große Erfahrung und ist für eine routinemäßige Anwendung nicht geeignet.

Intraoperative Autotransfusion(12, 22, 25) bedeutet die Rückgabe von Blut, das aus dem Operationsfeld abgesaugt wurde. Eine Retransfusion von derartigem Vollblut nach Antikoagulation und Filtration ist mit wenig aufwendigen Geräten möglich. Das Problem der intraoperativen Autotransfusion ist der Gehalt dieses Blutes an Antikoagulans, an Produkten aus traumatisiertem Gewebe und Hämolyse, an Gerinnungsaktivatoren, Enzymen und Mediatoren(28). Die damit verbundenen Gefahren lassen sich durch die Anwendung von Autotransfusionsgeräten umgehen, die eine Aufarbeitung des Blutes erlauben. Dabei werden durch Zentrifugation das hämolytische Plasma abgetrennt und verworfen, die Erythrozyten gewaschen, konzentriert und retransfundiert. Die durch intraoperative Autotransfusion rasch zur Verfügung gestellten Erythrozyten sind voll funktionsfähig und vital $(2,21,26)$. Nach dem Zellwaschvorgang sind Antikoagulans und unerwünschte Lyseprodukte in ausreichendem Maße entfernt. Wie bei der Verwendung von homologen Erythrozytenkonzentraten ist der Plasmaverlust zusätzlich zu ersetzen. Kontraindiziert ist eine intraoperative Autotransfusion bei bakterieller Kontamination oder bei Operation eines malignen $\mathrm{Tu}$ mors.

Bei der dorsalen Spondylodese kommen für die intraoperative Autotransfusion, wegen des großflächigen Gewebekontaktes des Blutes und der Verunreinigung mit Knochen- und Fettpartikeln sowie Spüllösung, nur Geräte mit Zellwaschvorgang in Betracht. Mehrere Berichte über die Anwendung intraoperativer Autotransfusion bei Skolioseoperationen liegen vor $(5,9,14,23)$.

Eine Retransfusion von Drainagenblut wird bisher nur bei Thoraxdrainagen nach Herzchirurgie durchgeführt, wo ei- ne gesonderte Antikoagulation meist entfällt(27). Eine zeitliche Begrenzung und eine Aufarbeitung dieses Blutes ist sinnvoll.

Die einzelnen Formen autologer Transfusion sind erfolgreich bei Skolioseoperationen eingesetzt worden $(5,8-10$, 14-17, 23, 32). Der Blutbedarf konnte dadurch jedoch nur zu einem Teil reduziert werden, nur in einzelnen Fällen konnte auf homologe Transfusion völlig verzichtet werden. Für eine effektivere Bluteinsparung erscheint daher eine Kombination der Autotransfusionsmethoden sinnvoll(9). Wir berichten über den kombinierten Einsatz von präoperativer Eigenblutspende, normovolämischer Hämodilution und intraoperativer Autotransfusion zur Bluteinsparung bei der operativen Behandlung idiopathischer Skoliosen.

\section{Methoden}

Zwischen Januar 1982 und Mai 1985 wurde an unserer Klinik in 89 Fällen eine dorsale Spondylodese mit dem Harrington-Instrumentarium zur Behandlung einer idiopathischen Skoliose durchgeführt. Über Operationsverfahren und Ergebnisse wird an getrennter Stelle berichtet. Bei 16 der Patienten wurde eine kontrollierte Hypotension mit Nitroglycerin durchgeführt (4). Bei 31 der Patienten wurde eine limitierte normovolämische Hämodilution angewandt (18). Dazu wurde nach Narkoseeinleitung über eine großlumige Kanüle $450 \mathrm{ml}$ Blut in einen Blutbeutel (Biopack $\left.^{\circledR}\right)^{*}$ mit CPD-Stabilisatorlösung abgenommen, unter gleichzeitiger Zufuhr von $500 \mathrm{ml} 6 \%$ iger Dextran 60 Lösung.

Bei 72 Patienten wurde eine intraoperative Autotransfusion mit dem Cell-Saver III ${ }^{\circledR * *}$ vorgenommen (22). Dabei wird über eine doppellumige Saugleitung Blut aus dem Operationsgebiet mit einem Antikoagulans gemischt (ca. 300 I.E. Heparin in $100 \mathrm{ml} \mathrm{NaCl} 0,9$ (pro $500 \mathrm{ml}$ Blut) und mit einem Sog von maximal $100 \mathrm{mmHg}$ in ein Reservoir gesaugt. Durch einen 120- $\mu \mathrm{m}$-Filter werden Knochensplitter, Gewebestücke und Thromben zurückgehalten. Nach Abzentrifugation des Plasmas werden die Erythrozyten mit einem Liter Kochsalzlösung automatisch gewaschen. Die gesamte Aufbereitung eines Erythrozytenkonzentrats benötigt ca. 7 Minuten.

Seit 1983 wurde zusätzlich ein Eigenblutspendeprogramm angeboten, an dem bis Mai 198537 Patienten teilnahmen. $\mathrm{Zu}$ jedem Abnahmetermin wurden nach Voruntersuchung und Laborwerten $450 \mathrm{ml}$ Blut in einen Doppelbeutel mit CPD-Stabilisatorlösung abgenommen (Fenwal $\left.{ }^{\circledR}\right)^{* * * *}$. Es wurden 2-5, am häufigsten 3 Spenden im Abstand von 1-6 Wochen durchgeführt. Eine orale Eisensubstitution wurde von der 1 . Spende ab bis über den Operationstermin hinaus mit Eisen(II)sulfat, $300 \mathrm{mg} / \mathrm{Tag}$ p.o., durchgeführt. Nach Zentrifugation wurde das Plasma, als fresh-frozenPlasma (FFP) bei $-40^{\circ} \mathrm{C}$, und die Erythrozyten nach der low-glycerol-Methode tiefgefroren (1). Die in der Gasphase des flüssigen Stickstoffs bei ca. $-165^{\circ} \mathrm{C}$ gelagerten Erythrozytenkonzentrate wurden am Operationstag im Wasserbad bei $45^{\circ} \mathrm{C}$ aufgetaut und das Glycerin durch Zentrifugation ausgewaschen (Cell Washer $115^{\circledR * *}$ bzw. manuell

\footnotetext{
* Biotest Pharma, Dreieich

** Haemonetics, München

$\because *$ Travenol Lab., Thetford
} 
Tab.1 Blutverlust und Transfusionen bei dorsalen Spondylodesen wegen idiopathischer Skoliose.

\begin{tabular}{|c|c|c|c|c|c|c|}
\hline & $\begin{array}{l}\text { intraop. } \\
\text { Blutverlust } \\
\text { (1) }\end{array}$ & $\begin{array}{l}\text { homologe } \\
\text { intraop. } \\
\text { (EK) }\end{array}$ & $\begin{array}{l}\text { ransfusion } \\
\text { intraop. + } \\
\text { (EK) }\end{array}$ & $\begin{array}{l}\text { postop. } \\
\text { (FFP) }\end{array}$ & $\begin{array}{l}\text { autologe } \mathrm{Tr} \\
\text { Cell Saver } \\
\text { (EK) }\end{array}$ & $\begin{array}{l}\text { רsfusion } \\
\text { Blutspende } \\
\text { (EK + FFP) }\end{array}$ \\
\hline $\begin{array}{l}\text { Gruppe I } \\
\text { ohne Autotransfusion }(n=17)\end{array}$ & $4,8 \pm 1,6$ & $5,8 \pm 0,5^{*}$ & $8,4 \pm 0,9^{*}$ & $5,1 \pm 1,5$ & - & - \\
\hline $\begin{array}{l}\text { Gruppe } \| \\
\text { intraoperative Autotransfusion }(n=35)\end{array}$ & $4,7 \pm 1,4$ & $2,7 \pm 0,3^{*}$ & $3,9 \pm 0,3^{*}$ & $4,2 \pm 2,1$ & $6,0 \pm 2,3$ & - \\
\hline $\begin{array}{l}\text { Gruppe III } \\
\text { präoperative Blutspende + intraoperative Auto- } \\
\text { transfusion }(n=37)\end{array}$ & $4,8 \pm 1,8$ & $0,4 \pm 0,2^{*}$ & $1,2 \pm 0,6^{*}$ & $1,0 \pm 0,4^{*}$ & $5,9 \pm 2,6$ & $3,1 \pm 0,8$ \\
\hline
\end{tabular}

Mittelwert $\pm s$

Medianwert $\pm s$
$\mathrm{EK}=$ Erythrozytenkonzentrat

$\mathrm{FFP}=$ fresh frozen plasma einmal Waschlösung, zweimal $\mathrm{NaCl}$-Lösung) und standen innerhalb von 1-3 Stunden zur Verfügung.

\section{Ergebnisse}

Es ergaben sich 3 Gruppen von Patienten:

Gruppe I: 17 Patienten, bei denen eine normovolämische Hämodilution, aber weder eine präoperative Eigenblutabnahme noch eine intraoperative Autotransfusion erfolgte.

Gruppe II: 35 Patienten, bei denen eine intraoperative Autotransfusion durchgeführt wurde.

Gruppe III: 37 Patienten, bei denen neben der intraoperativen Blutaufarbeitung auch Eigenblut aus präoperativen Spenden zur Verfügung stand. Die Gruppen waren im Alter der Patienten (16.8 $\pm 5 \mathrm{~J}$ in Gr. I, $17.5 \pm 8 \mathrm{~J}$ in Gr. II, $17.3 \pm 4 \mathrm{~J}$ in Gr. III) und Dauer der Operation (186 $\pm 42 \mathrm{~min}$ für Gr. I, $180 \pm 30 \mathrm{~min}$ für Gr. II, $174 \pm 34$ min für Gr. III) vergleichbar. Auch der intraoperative Blutverlust, bestehend aus gemessenem, abgesaugtem Blut und gemessenem oder geschätztem Blut in Tüchern und Tupfern, war nahezu identisch für alle 3 Gruppen (Tab. 1). Der Blutverlust zeigte eine breite Streuung, die auch mit zunehmender Zahl an Patienten nicht abnahm.

Um die Effizienz der intraoperativen Autotransfusion bei der dorsalen Spondylodese abzuschätzen wurde untersucht, welcher Teil des Blutverlustes für den Patienten zurückgewonnen werden kann. Die genaue Bilanzierung des Blutverlustes bei 20 Operationen durch Messung des abgesaugten Blutes, Wiegen von Tupfern und Tüchern und Berücksichtigung von Spüllösungen und Antikoagulans ergab, daß $68 \%$ ( \pm 12 ) des Gesamtblutverlustes im Sauger erschienen und damit für eine Autotransfusion verfügbar wurden. Ein Vergleich der Blutmengen und Hämatokritwerte in Saugerreservoir und Transfusionsbeutel zeigte, daß $81 \%$ ( \pm 10$)$ der angesaugten Erythrozyten den Patienten schließlich rücktransfundiert werden konnten. Das entspricht $0,68 \times 0,81=55 \%$ des Gesamtblutverlustes. Der Hämatokrit des angesaugten Blutes lag im Mittel bei $17 \%$, der des aufbereiteten Erythrozytenkonzentrats bei etwa $60 \%$. Im Durchschnitt wurden mit dem Cell-Saver pro Operation etwa 6 Portionen gewachsenes Erythrozytenkonzentrat zu je $240 \mathrm{ml}$ hergestellt (Tab. 1).

Die Einsparung an homologer Transfusion durch intraoperative Autotransfusion ist aus $\mathrm{Abb} .1 \mathrm{zu}$ ersehen, die die
Häufigkeitsverteilung von Transfusionen in den 3 Patientengruppen darstellt. Alle Transfusionen während des gesamten Krankenhausaufenthaltes wurden berücksichtigt. Durch intraoprative Autotransfusion werden die Werte deutlich zu niedrigerer Transfusionsanzahl verschoben. Die Menge an Transfusionen ist in Transfusionseinheiten und nicht in $\mathrm{ml}$ angegeben, da die wesentlichen Transfusionsrisiken weniger vom transfundierten Volumen, als von der Zahl der beteiligten Blutspender abhängen. Die intraoperative Autotransfusion reduzierte die durchschnittliche Anzahl an homologen Transfusionen von 8,4 (Gruppe I) auf 3,9 (Gruppe II), also um $54 \%$ (Tab. 1). Für die teilweise durchgeführte normovolämische Hämodilution oder kontrollierte Hypotension ergab sich kein signifikanter Effekt auf den Fremdblutverbrauch. Auch durch die Verringerung des Fremdblutbedarfes durch intraoperative Autotransfusion (Gruppe II) auf etwa die Hälfte konnte bei keinem der Patienten auf homologe Transfusion gänzlich verzichtet werden. Obgleich diese bei einem Patienten nur in Form von homologem FFP stattfand.

Den Patienten der Gruppe III standen aus präoperativen Eigenblutspenden zusätzlich im Schnitt 3 autologe EK und Eigenplasma zur Verfügung. Insgesamt kam es zu einer weiteren Verringerung an Transfusionen (Abb.1) und zu einer Vermeidung von homologer Transfusion durch Eigenblut aus präoperativer Spende und intraoperativer Blutaufbereitung. 15 von 37 Patienten der Gruppe III erhielten keine homologen Erythrozytenkonzentrate, 13 auch kein homologes Plasma. Die Gabe von Fremdblut reduzierte sich im Durchschnitt auf 1,2 Transfusionseinheiten, dies ist $1 / 7$ der ursprünglichen Menge (Tab. 1). Aufgrund des Eigenplasmas wurde auch der Bedarf an homologem FFP deutlich gesenkt. $2 / 3$ der noch nötigen Transfusionen erfolgten erst postoperativ, besonders am 2.-5. postoperativem Tag. $\mathrm{Zu}$ diesem Zeitpunkt zeigte der Verlauf der $\mathrm{Hb}$-Werte regelmäßig einen postoperativen weiteren Abfall mit den tiefsten Werten um den 4. Tag und einen anschließenden Anstieg. In Abb. 2 sind als Beispiele von 4 Patienten die Hb-Werte im Verlauf aufgetragen. Die präoperativen Werte zeigten einen Hb-Abfall, wenn zwischen den Blutabnahmen oder vor dem Operationstermin weniger als 10 Tage lagen (z. B. Pat. A. K. in Abb. 2). Waren die Zeiträume gröBer als 3-4 Wochen, so trat der postoperative Hb-Anstieg etwas später auf (z. B. Pat. B. R. in Abb. 2), als Hinweis, daß die Erythropoesestimulierung bis zum Operationstermin bereits abgeklungen war. 

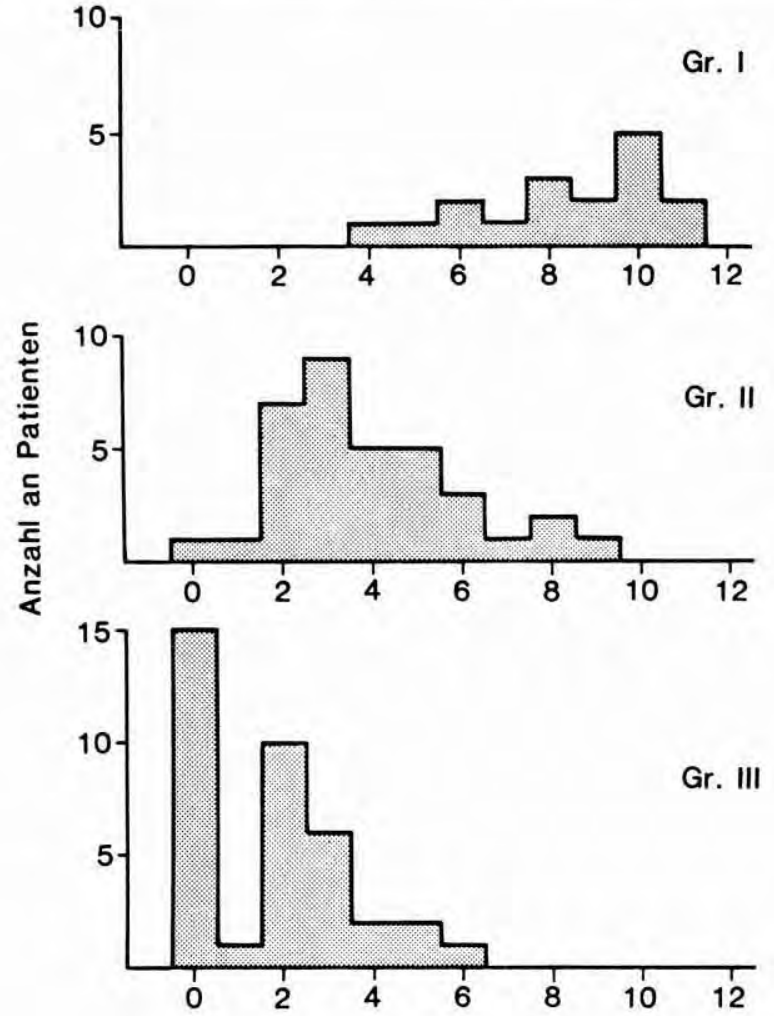

\section{Einheiten homologer Transfusion}

Abb.1 Häufigkeit homologer Transfusion bei dorsaler Spondylodese.

Gruppe I: ohne Autotransfusion

Gruppe II: mit intraoperativer Autotransfusion

Gruppe III: mit präoperativer Blutspende und intraoperativer Autotransfusion

Es wurden intra- und postoperativ keine Komplikationen beobachtet, die in irgendeinen Zusammenhang zur autologen Transfusion standen. Insbesondere wurden keine Gerinnungsstörungen und pulmonalen Störungen beobachtet, wie sie nach homologer Massentransfusion vorkommen. Hingegen fiel auf Intensiv- und Normalstation eine deutlich raschere postoperative Erholung auf.

\section{Diskussion}

Die dorsale Spondylodese unter Verwendung des Harrington-Instrumentariums zur Behandlung einer idiopathischen Skoliose ist ein großer Eingriff an jungen Patienten, der mit einem erheblichen Blutverlust einhergeht. Die Angaben in der Literatur darüber schwanken $(5,9,15,17$, 23,32 ) und sind abhängig vom operativen Vorgehen, von der Blutverlustschätzung, der Operationslagerung und der Flüssigkeitsbilanzierung. Die vorliegende Arbeit beschränkt sich auf Eingriffe bei idiopathischer Skoliose, andere Indikationen für dorsale Spondylodesen mit deutlich geringerem Blutverlust sind nicht enthalten. Die Messung des Blutverlustes ergab für das Blut in Tüchern und Tupfern einen häufig unterschätzten Anteil von etwa $1 / 3$. Die hier angestrebte positive Flüssigkeitsbilanz und die zum Teil durchgeführte Hämodilution führen nachweislich über eine verbesserte Mikrozirkulation zu einem höheren

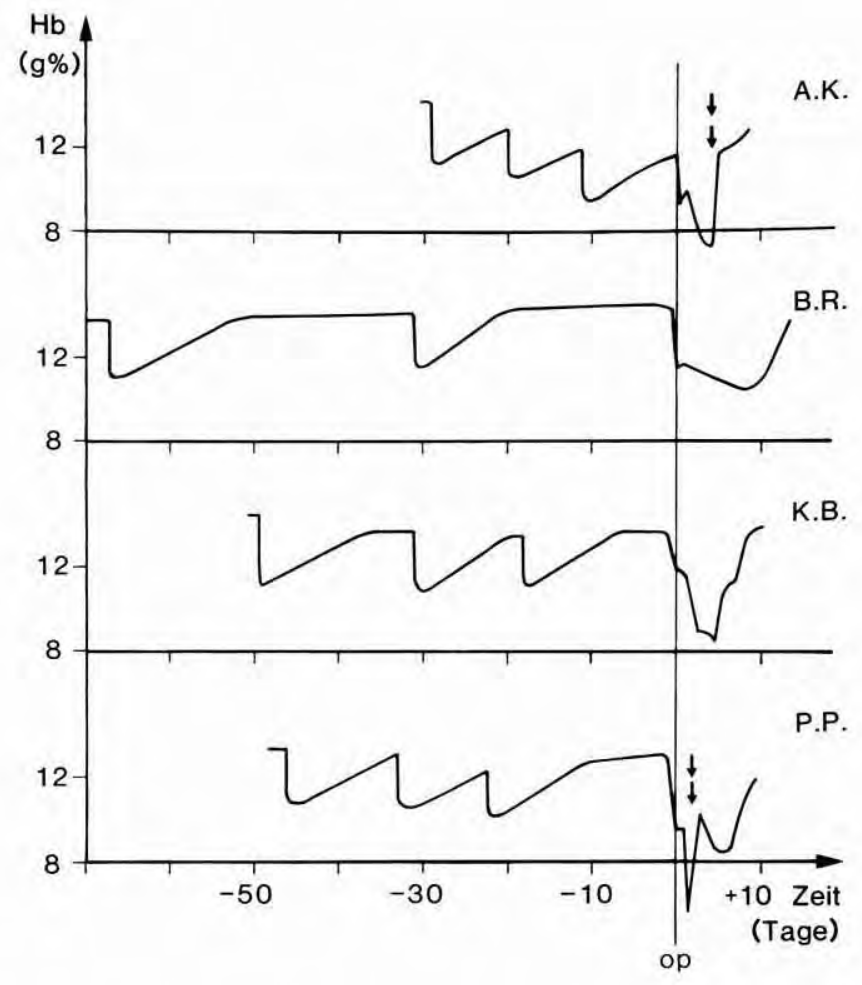

Abb. 2 Verlauf der Hämoglobinwerte von 4 Skoliosepatienten mit präoperativen Eigenblutspenden, dorsaler Spondylodese und intraoperativer Autotransfusion

Blutverlust in Form verdünnten Blutes, aber zu geringeren postoperativen Blutungen $(4,18)$. Letztlich hat sich jedes Autotransfusionsprogramm nach dem jeweiligen Gegebenheiten des Blutverbrauches auszurichten.

Während die Angaben über den Blutverlust auseinandergehen, wird übereinstimmend beschrieben, daß die Operation regelmäßig Transfusionen nötig macht, und, daß die Anwendung einer einzelnen Autotransfusionsmethode den Fremdblutbedarf verringern, aber nicht beseitigen kann $(10,15,23)$. Wichtig erscheint es, den postoperativen Blutbedarf in die Betrachtung mit einzubeziehen, ebenso wie die Gabe von Fremdplasma, daß die Risiken der Infektionsübertragung und der Sensibilisierung gleichermaßen beinhaltet.

Durch maschinelle intraoperative Autotransfusion können abgesaugte Erythrozyten ohne Antikoagulans und ohne unerwünschte Produkte aus Hämolyse und Gewebstraumatisierung den Patienten zurückgegeben werden. Eine Ausbeute von über $80 \%$, die deutlich über früheren Berichten $(5,9)$ liegt, ist möglich, wenn Hämolyse durch Luftansaugung oder zu hohem Sog sorgfältig vermieden wird. Damit konnte über die Hälfte des intraoperativen Gesamtblutverlustes dem Patienten erhalten werden, wie es auch bei anderen Operationen, z. B. in der Gefäßchirurgie, beobachtet wird (11). Diese intraoperative Bluteinsparung geht mit einer deutlichen, ebenso hohen Einsparung an ho- 
Tab. 2 Vorteile der autologen Transfusion

$\begin{array}{ll}\text { Keine Krankheitsübertragung } & \text { Hepatitis, AIDS, Cytomegalie, } \\ \text { Malaria, Syphilis, u. a. } \\ \text { Keine Unverträglichkeits- } & \text { hämolytisch, febril, allergisch, } \\ \text { reaktion } & \text { G.v.H. } \\ \text { Keine Allo-Sensibilisierung } & \text { gegen Blutgruppen-, Histokom- } \\ & \text { patibilitäts- und Plasmaantigene } \\ \text { Verfügbarkeit } & \text { auch bei seltener Blutgruppe, ir- } \\ & \text { regulären Antikörpern, } \\ \text { Entlastung der Blutdepots } & \text { bei steigendem Bedart/ } \\ & \text { sinkender Spendebereitschaft } \\ \text { Kostenersparnis } & \text { u.a. entfällt Kreuzprobe und An- } \\ & \text { tikörpersuchtest } \\ \text { (bei intraop. Autotransfusion ab } & 2 \text { EK) } \\ \text { hohe Qualität, keine Lagerungs- } & \text { 24-Std.-Überlebenszeit, 2,3- } \\ \text { schäden } & \text { DPG, p50 } \\ & \text { (bei Tiefgefrierlagerung) } \\ \text { Keine religiösen Einwände } & \text { Zeugen Jehovas } \\ & \text { (nicht bei präop. Blutspende) } \\ \text { (bei präoperativer Blutspende) }\end{array}$

mologer Transfusion einher. Nicht nur der Anteil an autologem Blut an der gesamten Transfusionsmenge, sondern vielmehr der direkte Vergleich der Anzahl homologer Transfusionen zur Kontrollgruppe zeigt dies an (Tab. 1). Ähnliche Ergebnisse haben Paravichini und Mitarb. (23) beschrieben, die bei dorsalen Spondylodesen, durchgeführt an der Orthopädischen Universitätsklinik Münster, bei einem mittleren Blutverlust von 4,5 Liter durch intraoperative Autotransfusion den Bedarf an homologen Erythrozyten auf $900 \mathrm{ml}$, entsprechend etwa 3,5 Transfusionseinheiten, reduzieren konnten.

Durch intraoperative Autotransfusion allein gelingt es nicht, den Skoliosepatienten homologe Transfusionen und ihre Risiken gänzlich zu ersparen. Zusätzliches Eigenblut kann in ausreichendem Maße nur durch präoperative Eigenblutspende bereitgestellt werden. Während von Blutspendern wiederholte wöchentliche Abnahmen von $500 \mathrm{ml}$ ohne $\mathrm{Hb}$-Abfall kompensiert werden(7) und während die Plasmaproteine rasch nachgebildet sind (24), benötigen die jugendlichen Skoliosepatienten - die jüngste Spenderin war 12 Jahre alt - nach unseren Befunden 10 bis 14 Tage, um ihren ursprünglichen $\mathrm{Hb}$-Wert wieder zu erreichen. Zur Ansammlung der notwendigen Blutkonservenzahl können diese Zeiten nur mit Tieffrierlagerung der Erythrozyten eingehalten werden. Der weitere wesentliche Vorteil ist das anfallende Eigenplasma, das als fresh-frozen-Plasma den bei diesem Blutverlust notwendigen Ersatz von Gerinnungsfaktoren erlaubt.

Ein Hb-Abfall am 2.-4. postoperativen Tag wird bei Operationen mit derartigem Blutverlust regelmäßig beobachtet, unabhängig von autologer Transfusion. Wenn er verdünnungsbedingt durch Flüssigkeitsverschiebungen zustandekommt und, weil das Plasma schneller nachgebildet wird als die Erythrozyten (7), und weniger durch postoperativen Blutverlust ins Gewebe(32), so ist abzuwägen, ob dadurch eine Transfusion wirklich notwendig wird. Für die Effektivität eines Autotransfusionsprogrammes ist es wesentlich, $\mathrm{da}$ dieser Verlauf bekannt ist und, daß auch postoperativ auf der Station homologe Transfusion kritisch eingesetzt wird.

Durch die Kombination von präoperative Blutspende und intraoperativer Autotransfusion konnten im Durchschnitt jedem Patienten 7,2 Erythrozytenkonzentrate und 4,1 fresh-frozen-Plasma eingespart werden, und in vielen Fällen ein Verzicht auf jegliche homologe Transfusion erreicht werden. Damit werden Transfusionsrisiken, die im Falle dieser jungen Patientin als besonders schwerwiegend anzusehen sind ausgeschlossen. Bleibende Schäden nach einer etwaigen Hepatitis oder durch die Alloimmunisierung entstehende Komplikationen bei späteren Transfusionen sollten unbedingt vermieden werden. Auch für spätere Schwangerschaften - $83 \%$ der Patienten waren junge Mädchen - stellt jede homologe Transfusion durch die Sensibilisierung gegen Blut- und Gewebsantigene eine Gefährdung dar (13).

Neben der Vermeidung dieser Risiken bietet autologes Blut weitere Vorteile (Tab. 2). Mehrfach ist die Kosteneffizienz der autologen Transfusionsmethoden aufgezeigt worden $(22,25,30)$. Aufwand und Kosten der ErythrozytenTieffrierung werden noch weitgehend überschätzt, doch nimmt die Verbreitung dieser Methode laufend zu. Spezielle Vorteile der präoperativen Blutspende sind die Stimulierung der Erythropoese, welche die postoperative Erholung beschleunigt, wie auch die große Motivation der Patienten, die an ihrer Operationsvorbereitung aktiv mitwirken können.

Vor allem aber ist die Qualität autologen Blutes hervorzuheben. Sowohl tiefgefrorene gelagerte Erythrozyten(31), als auch intraoperativ aufbereitete zeigen nach Transfusion normale Überlebenszeiten(2). Ebenso entsprechen sie in ihrem Gehalt an 2,3-DPG und damit in ihrer Sauerstoffbindungscharakteristik frischen Erythrozyten(21). Dieser deutliche Unterschied in der Funktionstüchtigkeit gegenüber gelagerten Fremdblutkonserven ist selbst nach Transfusion im Patienten noch nachweisbar(26). Die auffallend rasche Erholung nach Skolioseoperationen bei Patienten die autologes Blut erhalten haben, im Vergleich zu solchen mit einer entsprechenden homologen Massivtransfusion, wird ihren Grund in diesem Unterschied in der Qualität haben.

So ist das hier aufgezeigte Autotransfusionsprogramm bei operativer Behandlung idiopathischer Skoliosen ein Beispiel, wie bei elektiven Eingriffen mit erheblichem Blutverlust durch die Kombination verschiedener Arten autologer Transfusion und enge Zusammenarbeit von Patient, Operateur, Anästhesist, Stationsarzt, Transfusions- und Intensivmediziner Risiken für die Patienten vermieden werden können und eine optimale Hämotherapie möglich wird.

\section{Literatur}

(1) Akerblom, O., C. F. Högman:

Frozen blood: A method for lowglycerol, liquid nitrogen freezing allowing different post-thaw deglycerolization procedures. Transfusion 14 (1975) 16

(2)Ansell, J., N. Parrilla, M. King, L. Fournier, I. Szymanski, P. Doherty, T. V. Salm, B. Cutler: Survival of autotransfused red blood cells recovered from the surgical field during cardivascular operations. J. Thorax. Cardiovasc. Surg. 84 (1982) 387

(3) Ascari, W. Q., P. C. Jolly, P. A Thomas: Autologous blood transfusion in pulmonary surgery. Transfusion 8 (1968) 111
(4) Barbier-Böhm, G., J. M. Desmonds, E. Conderc, D. Moulin, P. Prokorimer: Comparative effects of induced hypotension and normovolemic hemodilution on bloodloss in hip surgery. Eur. Acad. Anaesth. 14 (1981) 103

(5)Bonnet, C. A., R. Lapin, J. Giddings: Intraoperative autologous transfusion in orthopaedics. Orthop. Rev. 10 (1981) 49

(6) Brzica, S. M., A. A. Pineda, H. F. Taswell: Autologous blood transfusion. Mayo Clin. Proc. 51 (1976) 723

(7) Coleman, D. H., A. R. Stevens, H. D. Dodge, C. A. Finch: Rate of blood regeneration after blood loss. Arch. Int. Med. 92 (1953) 341 
(8)Cowell, H. R., J. W. Swickard: Autotransfusion in children's orthopaedics. J. Bone Joint Surg. 56 (1974) 908

(9) Flynn, J. C., T. S. Csencsitz: Present status of intraoperative blood recovery during orthopaedic surgery. Jefferson Orthop.J. 8 (1980) 22 (10)Fricke, M., V. Kretschmer: Die Eigenblutspende in Bocksprungtechnik. Beitr. Infusionstherapie klin. Ernährung, Karger, Basel 15 (1986) 19 (11) Hansen, E.: Autotransfusion in der Gefäßchirurgie. In: „Anaesthesiologische Probleme in der Gefäß chirurgie." Hrsg. von Martin, E., F. Jesch und K. Peter, Anaesth. Intensivmed. Bd. 177, Springer, Berlin 1985, S. 171

(12) Hansen, E.: Bedeutung der IAT in der operativen Medizin. In: „Hämodilution und Autotransfusion in der perioperativen Phase", hrsg. von Lawin, P. und D. Paravicini, Intensivmed. Notfallmed. Anästhesiol. Bd. 49 Thieme, Stuttgart 1984 , S. 49

(13) Kretschmer, V., D. Koßmagk: Bedeutung der Blutgruppen für die Bluttransfusion. Lab, med. 8 (1984) 14

(14)Kruger, L. M., J. M. Colbert: Intraoperative autologous transfusion in children undergoing spinal surgery. J. Ped. Orthop. 5 (1985) 30 (15) Mandel, R. J., M.D. Brown, N.C. McCollough, V.Pallares, R. Varlotta: Hypotensive anesthesia and autotransfusion in spinal surge- ry. Clin. Orthop. Res. 154 (1981)

(16) Mann, M., H. J. Sachs, D. Goldfinger: Safety of autologous blood donation prior to elective surgery for a variety of potentially ,high-risik" patients. Transfusion 23 (1983 229

(17) Martin, E., E. Ott: Extreme hemodilution in the Harrington procedure. Biblthca haemat. 47 (1981) 322

(18) Martin, E., E. Ott: Die praktische Anwendung der isovolämischen Hämodilution. In: „Hämodilution und Autotransfusion in der perioperativen Phase“, hrsg. von Lawin, P. und D. Paravicini, Intensivmed. Notfallmed. Anästhesiol. Bd. 49. Thieme, Stuttgart 1984, S. 37

(19) Matzen, K. A.: Ergebnisse der operativen Skoliosebehandlung an der Orthopädischen Klinik München. Z. Orthop. 121 (1982) 292

(20) Müller-Eckhardt, C.: Gefahren bei der Transfusionstherapie. Anästh. Intensivther. Notfallmed. 15 (1980) 179

(21) Orr, M.: Autotransfusion: The use of washed red cells as an adjunct to component therapy. Surgery 84(1978) 728

(22) Paravicini, D., P. Lawin: Intraoperative Autotransfusion - gestern heute, morgen. Anästh. Intensivmed. 24 (1983) 137

(23) Paravicini, D., R. Frisch, B. Stinnesbeck, P. Lawin: Intraoprative Au- totransfusion bei großen orthopädischen Eingriffen. Z. Orthop. 12 (1983) 278

(24) Parlett, R. C., R. Naidu: Serial serum protein determinations in scoliotic children treated by spine fusion and autotransfusion. J. Bone Joint Surg. 61 (1979) 105

(25) Popovsky, M. A., P. A. Devine, H. F. Taswell: Intraoperative autologous transfusion. Mayo Clin. Proc 60 (1985) 125

(26) Reinhart, K., C. Wiegand, T. Kersting: Zur Veränderung der Sauerstoffaffinität durch maschinelle Autotransfusion. In: „Hämodilution und Autotransfusion in der perioperativen Phase", hrsg. von Lawin, P. und D. Paravicini, Intensivmed. Notfallmed. Anästhesiol. Bd. 49, Thieme, Stuttgart 1984 , S. 115

(27) Schaff, H. V., J. M. Hauer

W. R. Bell: Retransfusion of shed mediastinal blood following cardiac surgery: a randomized, prospective study, J. Thoraxix Cardiovasc. Surg. 74 (1978) 4

Dr. med., Dr. rer. nat. E. Hansen Institut für Anästhesiologie der Universität München Klinikum Großhadern Postfach 701260 D-8000 München 70
(28) Stillmann, R. M., W. W. Wrezlewicz, B. Stanczewski, L. Chapa, M. J. Fox, P. N. Sawyer: The haematological hazards of autotransfusion. $\mathrm{Br}$. J. Surg. 63 (1976) 651

(29) Sunder-Plassmann, L., W. P. Klövekorn, K. Holpe, K. Messmer. Präoperative Hämodilution: Grundlagen, Adaptationsmechanismen und Grenzen klinischer Anwendung. Anaesthesist 25 (1976) 124

(30)Thurer, R. L., J. M. Hauer: Autotransfusion and blood conservation. Curr. Probl. Surg. 19 (1982) 98

(31) Valeri, C. R.: Factors influencing the 24-hour posttransfusion survival and the oxygen transport function of previously frozen red cells preserved with 40 per cent $\mathrm{w} / \mathrm{v}$ glycerol and frozen at $-80^{\circ} \mathrm{C}$. Transfusion 14 (1974) 1

(32) Viviani, G. R., J.T. S. Sadler, G. K. Ingham: Autotransfusion in scoliosis surgery. Clin. Orthop. Res. $135(1978) 74$ 